\title{
Extending Diabetic Retinopathy Imaging from Color to Spectra
}

\author{
Pauli Fält ${ }^{1}$, Jouni Hiltunen ${ }^{1}$, Markku Hauta-Kasari ${ }^{1}$, Iiris Sorri ${ }^{2}$, \\ Valentina Kalesnykiene ${ }^{2}$, and Hannu Uusitalo ${ }^{2,3}$ \\ 1 InFotonics Center Joensuu, Department of Computer Science and Statistics, \\ University of Joensuu, P.O. Box 111, FI-80101 Joensuu, Finland \\ \{pauli.falt, jouni.hiltunen, markku.hauta-kasari\}@ifc.joensuu.fi \\ http://spectral.joensuu.fi \\ 2 Department of Ophthalmology, Kuopio University Hospital and University of \\ Kuopio, P.O. Box 1777, FI-70211 Kuopio, Finland \\ iiris.sorri@kuh.fi, valentina.kalesnykiene@uku.fi \\ 3 Department of Ophthalmology, Tampere University Hospital, Tampere, Finland \\ hannu.uusitalo@uta.fi
}

\begin{abstract}
In this study, spectral images of 66 human retinas were collected. These spectral images were measured in vivo from 54 voluntary diabetic patients and 12 control subjects using a modified ophthalmic fundus camera system. This system incorporates the optics of a standard fundus microscope, 30 narrow bandpass interference filters ranging from 400 to 700 nanometers at $10 \mathrm{~nm}$ intervals, a steady-state broadband lightsource and a monochrome digital charge-coupled device camera. The introduced spectral fundus image database will be expanded in the future with professional annotations and will be made public.
\end{abstract}

Keywords: Spectral image, human retina, ocular fundus camera, interference filter, retinopathy, diabetes mellitus.

\section{Introduction}

Retinal image databases have been important for scientists developing improved pattern recognition methods and algorithms for the detection of retinal structures - such as vascular tree and optic disk - and retinal abnormalities (e.g. microaneurysms, exudates, drusens, etc.). Examples of such publicly available databases are DRIVE [1,2] and STARE [3. Also, retinal image databases including markings made by eye care professionals exist: e.g. DiaRetDB1 [4].

Traditionally, these databases contain only three-channel RGB-images. Unfortunately, the amount of information in images with only three channels is very limited (red, green and blue channel). In an RGB-image, each channel is an integrated sum over a broad spectral band. Thus, depending on application, an RGB-image can contain useless information that obscures the actual desired data. Better alternative is to take multi-channel spectral images of the retina, because with different wavelengths, different objects of the retina can be emphasized and researchers 
have indeed started to show growing interest in applications based on spectral color information. Fundus reflectance information can be used in various applications: e.g. in non-invasive study of the ocular media and retina [5]6], retinal pigments 8910, oxygen saturation in the retina 1112131415, etc.

For example, Styles et al. measured multi-spectral images of the human ocular fundus using an ophthalmic fundus camera equipped with a liquid crystal tunable filter (LCTF) [16. In their approach, the LCTF-based spectral camera measured spectral color channels from 400 to $700 \mathrm{~nm}$ at $10 \mathrm{~nm}$ intervals. The constant unvoluntary eye movement is problematic, since the LCTF requires separate lengthy non-stop procedures to acquire exposure times for the color channels and to perform the actual measurement. In general, human ocular fundus is a difficult target to measure in vivo due to the constant eye movements, optical aberrations and reflections from the cornea and optical media (aqueous humor, crystalline lens, and vitreous body), possible medical conditions (e.g. cataract), and the fact that the fundus must be illuminated and measured through a dilated pupil.

To overcome the problems of non-stop measurements, Johnson et al. introduced a snapshot spectral imaging apparatus which used a diffractive optical element to separate a white light image into several spectral channel images [17. However, this method required complicated calibration and data post-processing to produce the actual spectral image.

In this study, an ophthalmic fundus camera system was modified to use 30 narrow bandpass interference filters, an external steady-state broadband lightsource and a monochrome digital charge-coupled device (CCD) camera. Using this system, spectral images of 66 human ocular fundi were recorded. The voluntary human subjects included 54 persons with abnormal retinal changes caused by diabetes mellitus (diabetic retinopathy) and 12 non-diabetic control subjects. Subject's fundus was illuminated with light filtered through an interference filter and an 8-bit digital image was captured from the light reflected from the retina. This procedure was repeated using each of the 30 filters one by one. Resulting images were normalized to a unit exposure time and registered using an automatic GDB-ICP algorithm by Stewart et al. 18,19. The registered spectral channel images were then "stacked" into a spectral image. The final 66 spectral retinal images were gathered in a database which will be further expanded in the future. In the database, the 12 control spectral images are necessary for identifying normal and abnormal retinal features. Spectra from these images could be used, for example, as a part of a test set for an automatic detection algorithm.

The ultimate goal of the study was to create a spectral image database of diabetic ocular fundi with additional annotations made by eye care professionals. The database will be made public for all researchers, and it can be used e.g. for teaching, or for creating and testing new and improved methods for manual and automatic detection of diabetic retinopathy. To authors' knowledge, similar public spectral image database with professional annotations does not yet exist. 


\section{Equipment and Methods}

\subsection{Spectral Fundus Camera}

An ophthalmic fundus camera system is a standard tool in health care systems for the inspection and documentation of the ocular fundus. Normally, such system consists of xenon flash light source, microscope optics for guiding the light into the eye, and optics for guiding the reflected light to a standard RGB-camera. For focusing, there usually exists a separate aiming-light and a video camera.

In this study, a Canon CR5-45NM fundus camera system (Canon, Inc.) was modified for spectral imaging (see Figs. 1] and 2). All unneeded components of the system (including the internal light source) were removed - only the basic fundus microscope optics were left inside the device body - and appropriate openings were cut for the filter holders and the fiber optic cable. Four filter holders and a rail for them were fabricated from acrylic glass, and the rail was installed inside the fundus camera body. Each of the four filter holders could hold up to eight filters and the 30 narrow bandpass interference filters (Edmund Optics, Inc.) were attached to them in a sequence from 400 to $700 \mathrm{~nm}$ leaving the two last of the 32 positions empty. The transmittances of the filters are shown in Fig. 3 .

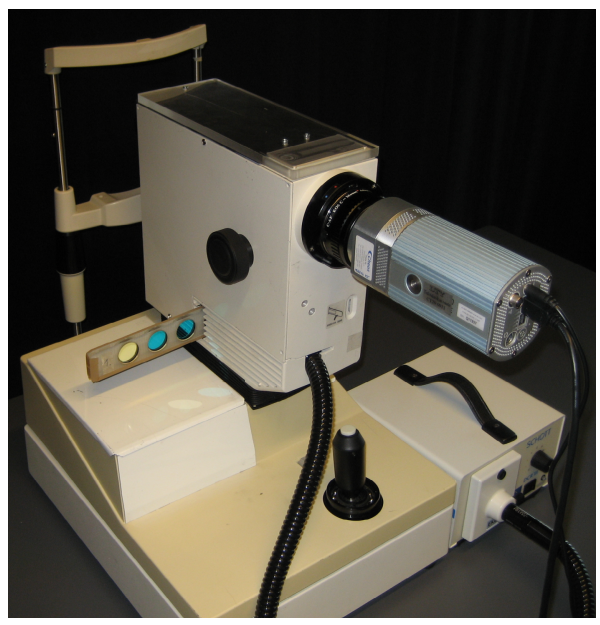

Fig. 1. The modified fundus camera system used in this study

The rail and the identical openings on both sides of the fundus camera allowed the filter holders to be slided through the device manually. A spring-based mechanical stopper locked the holder (and a filter) always in the correct place on the optical path of the system. As a broadband light source, an external Schott Fostec DCR III lightbox (SCHOTT North America, Inc.) with a 150 W OSRAM halogen lamp (OSRAM Corp.) and a daylight-simulating filter was used. Light 


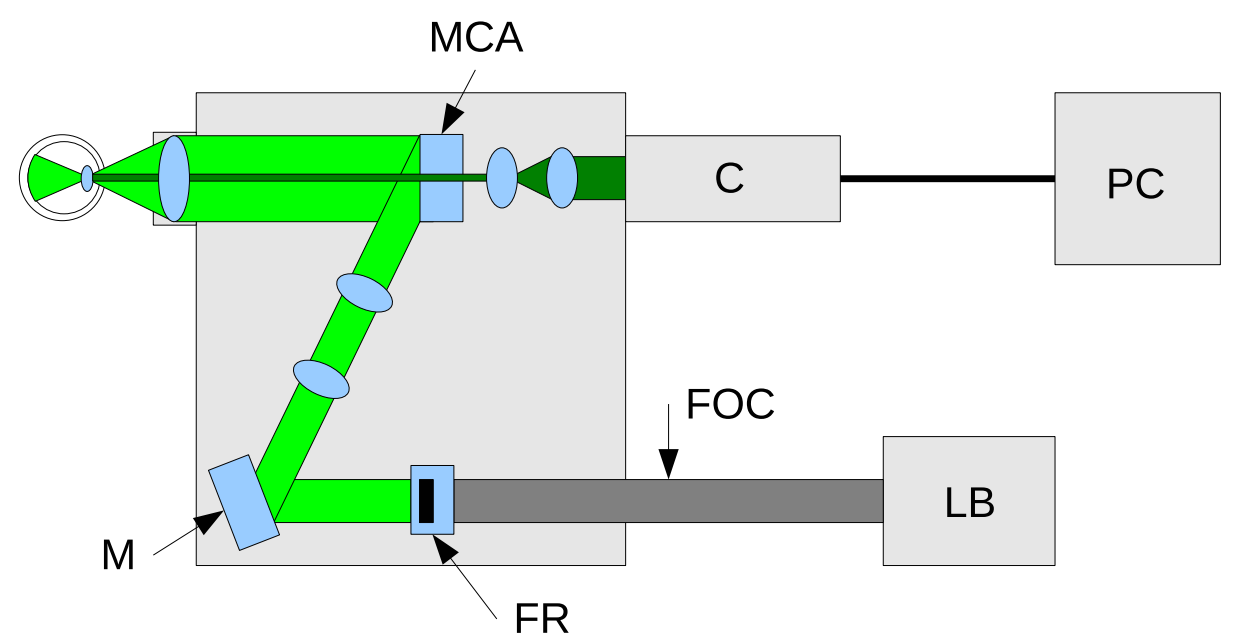

Fig. 2. Simplified structure and operation of the modified ophthalmic fundus camera in Fig. 1 a light box $(L B)$, a fiber optic cable $(F O C)$, a filter rail $(F R)$, a mirror $(M)$, a mirror with a central aperture $(M C A)$, a CCD camera $(C)$, a personal computer $(P C)$, and lenses (ellipses)

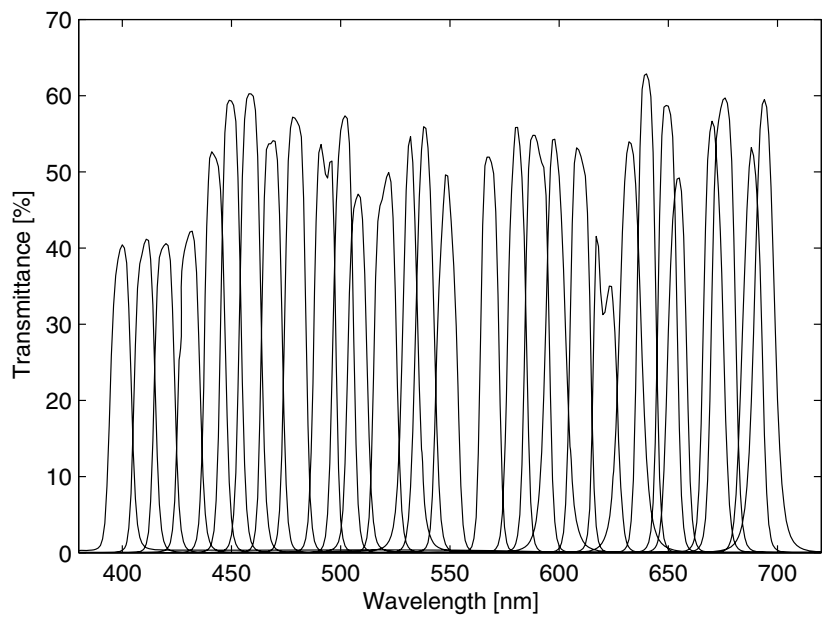

Fig. 3. The spectral transmittances of the 30 narrow bandpass interference filters

was guided into the fundus camera system via a fiber optic cable of the Schott lightbox. In the same piece as the rail was also a mount for the optical cable, which held the end of the cable tightly in place. The light source was allowed to warm up and stabilize for 30 minutes before the beginning of the measurements.

The light exiting the cable was immediately filtered by narrow bandpass filter and the filtered light was guided inside the subject's eye through a dilated 
pupil. Light reflecting back from the retina was captured with a QImaging Retiga4000RV digital monochrome CCD camera (QImaging Corp.), which had a $2048 \times$ 2048 pixel detector array and was attached to the fundus camera with a C-mount adapter. The camera was controlled via a Firewire port with a standard desktop PC running QImaging's QCapture Pro 6.0 software. The live preview function of the software allowed the camera-operator to monitor the subject's ocular fundus in real time, which was important for positioning and focusing of the fundus camera, and also for determining the exposure time. Exposure times were calculated from a small area in the retina with the highest reflectivity (typically the optic disk). The typical camera parameters - gain, offset and gamma - were set to 6,0 and 1, respectively. Gain-value was increased to shorten the exposure time.

The camera was programmed to capture five images as fast as possible and to save the resulting images to the PC's harddrive automatically. Five images per filter were needed because of the constant involuntary movements of the eye: usually at least one of the images was acceptable; if not, a new new set of five images was taken. Image acquisition produced 8-bit grayscale TIFF-images sized $1024 \times 1024$ pixels (using $2 \times 2$ binning). For each of the 30 filters, a set of five images were captured, and from each set only one image was selected for spectral image formation.

The selected images were co-aligned using the efficient automatic image registration algorithm by Stewart et al. called the generalized dual-bootstrap iterative closest point (GDB-ICP) algorithm 1819]. Some difficult image pairs had to be registered manually with MATLAB's Control Point Selection Tool [20]. The registered spectral channel images were then normalized to unit exposure time, i.e. 1 second, and stacked in wavelength-order into a $1024 \times 1024 \times 30$ spectral image.

\subsection{Spectral Image Corrections}

Let us derive a formula for the reflectance spectrum $\boldsymbol{r}_{\text {final }}$ at point $(x, y)$ in the final registered and white-corrected reflectance spectral image: The digital signal output $v_{i}$ for the interference filter $i, i=1, \ldots, 30$, from one pixel $(x, y)$ of the one-sensor CCD detector array is of the form

$$
v_{i}=\int_{\lambda} s(\lambda) t_{i}(\lambda) t_{\mathrm{FC}}(\lambda) t_{\mathrm{OM}}^{2}(\lambda) r_{\text {retina }}(\lambda) h_{\mathrm{CCD}}(\lambda) d \lambda+n_{i}
$$

where $s(\lambda)$ is the spectral power distribution of the light coming out of the fiber optic cable, $\lambda$ is the wavelength of the electromagnetic radiation, $t_{i}(\lambda)$ is the spectral transmittance of the $i$ th interference filter, $t_{\mathrm{FC}}(\lambda)$ is the spectral transmittance of the fundus camera optics, $t_{\mathrm{OM}}(\lambda)$ is the spectral transmittance of the ocular media of the eye, $r_{\text {retina }}(\lambda)$ is the spectral reflectance of the retina, $h_{\mathrm{CCD}}(\lambda)$ is the spectral sensitivity of the detector, and $n_{i}$ is noise. In Eq. (1), the second power of $t_{\mathrm{OM}}(\lambda)$ is used, because reflected light goes through these media twice.

Let us write the above spectra for pixel $(x, y)$ as discrete $m$-dimensional vectors (in this application $m=30) \boldsymbol{s}, \boldsymbol{t}_{i}, \boldsymbol{t}_{\mathrm{FC}}, \boldsymbol{t}_{\mathrm{OM}}, \boldsymbol{r}_{\text {retina }}, \boldsymbol{h}_{\mathrm{CCD}}$ and $\boldsymbol{n}$. Now, 
from (1) one gets the spectrum $\boldsymbol{v}$ for each pixel $(x, y)$ in the non-white-corrected spectral image as a matrix-equation

$$
\boldsymbol{v}=\boldsymbol{W} \boldsymbol{T}_{\mathrm{OM}}^{2} \boldsymbol{r}_{\text {retina }}+\boldsymbol{n}
$$

where $\boldsymbol{W}=\operatorname{diag}(\boldsymbol{w})$,

$$
\boldsymbol{w}=\boldsymbol{S} \boldsymbol{T}_{\mathrm{FC}} \boldsymbol{H}_{\mathrm{CCD}} \boldsymbol{T}_{\text {filters }} \mathbf{1}_{30}
$$

and $\boldsymbol{T}_{\mathrm{OM}}=\operatorname{diag}\left(\boldsymbol{t}_{\mathrm{OM}}\right), \boldsymbol{S}=\operatorname{diag}(\boldsymbol{s}), \boldsymbol{T}_{\mathrm{FC}}=\operatorname{diag}\left(\boldsymbol{t}_{\mathrm{FC}}\right), \boldsymbol{H}_{\mathrm{CCD}}=\operatorname{diag}\left(\boldsymbol{h}_{\mathrm{CCD}}\right)$, and $\boldsymbol{T}_{\text {filters }}$ is a matrix that has the spectra $\boldsymbol{t}_{i}$ on its columns. Finally, $\mathbf{1}_{30}$ denotes a 30-vector of ones.

Here $\boldsymbol{w}$ is a 30 -vector that describes the effect of the entire fundus imaging system, and it was measured by using a diffuse non-fluorescent Spectralon white reflectance standard (Labsphere, Inc.) as a imaging target instead of an eye. In this case

$$
\boldsymbol{v}_{\text {white }}=\boldsymbol{W} \boldsymbol{r}_{\text {white }}+\boldsymbol{n}_{\text {white }} .
$$

Spectralon-coating reflects $>99 \%$ of all the wavelengths in the visual range $(380$ $780 \mathrm{~nm})$. Hence, by assuming the reflectance $\boldsymbol{r}_{\text {white }}(\lambda) \approx 1, \forall \lambda \in[380,780] \mathrm{nm}$ in (41), and that the backround noise is minimal, i.e. $\boldsymbol{n} \approx \boldsymbol{n}_{\text {white }} \approx \mathbf{0}_{30}$, one gets (3). Now, (2) and (3) yield

$$
\boldsymbol{r}_{\text {final }}=\boldsymbol{T}_{\text {OM }}^{2} \boldsymbol{r}_{\text {retina }}=\boldsymbol{W}^{-1} \boldsymbol{v} .
$$

As usual, the superscript -1 denotes matrix (pseudo)inverse. In Eq. (5), $\boldsymbol{r}_{\text {final }}$ describes the "pseudo-reflectance" of the retina at point $(x, y)$ of the spectral image, because, in practice, it is not possible to measure the transmittance of the ocular media $t_{\mathrm{OM}}(\lambda)$ in vivo. One gets $\boldsymbol{W}$ and $\boldsymbol{v}$ by measuring the white reflectance sample and the actual retina with the spectral fundus camera, respectively. Another thing to consider is that a fundus camera is designed to take images of a curved surface, but no appropriate curved white reflectance standards exist. The Labsphere standard used in this study was flat, so the light was unevenly distributed on its surface. Because of this, using the 30 spectral channel images taken from the standard to make the corrections directly would have resulted in unrealistic results. Instead, a mean-spectrum from a $100 \times 100$ pixel spatial area in the middle of the white standard's spectral image was used as $\boldsymbol{w}$.

\section{Voluntary Human Subjects}

Using the spectral fundus camera system described above, spectral images of 66 human ocular fundi were recorded in vivo from 54 diabetic patients and 12 healthy volunteers. This study was approved by the local ethical committee of the University of Kuopio and was designed and performed in accordance with the ethical standards of the Declaration of Helsinki. Fully informed consent was obtained from each participant prior to his or her inclusion into the study. 


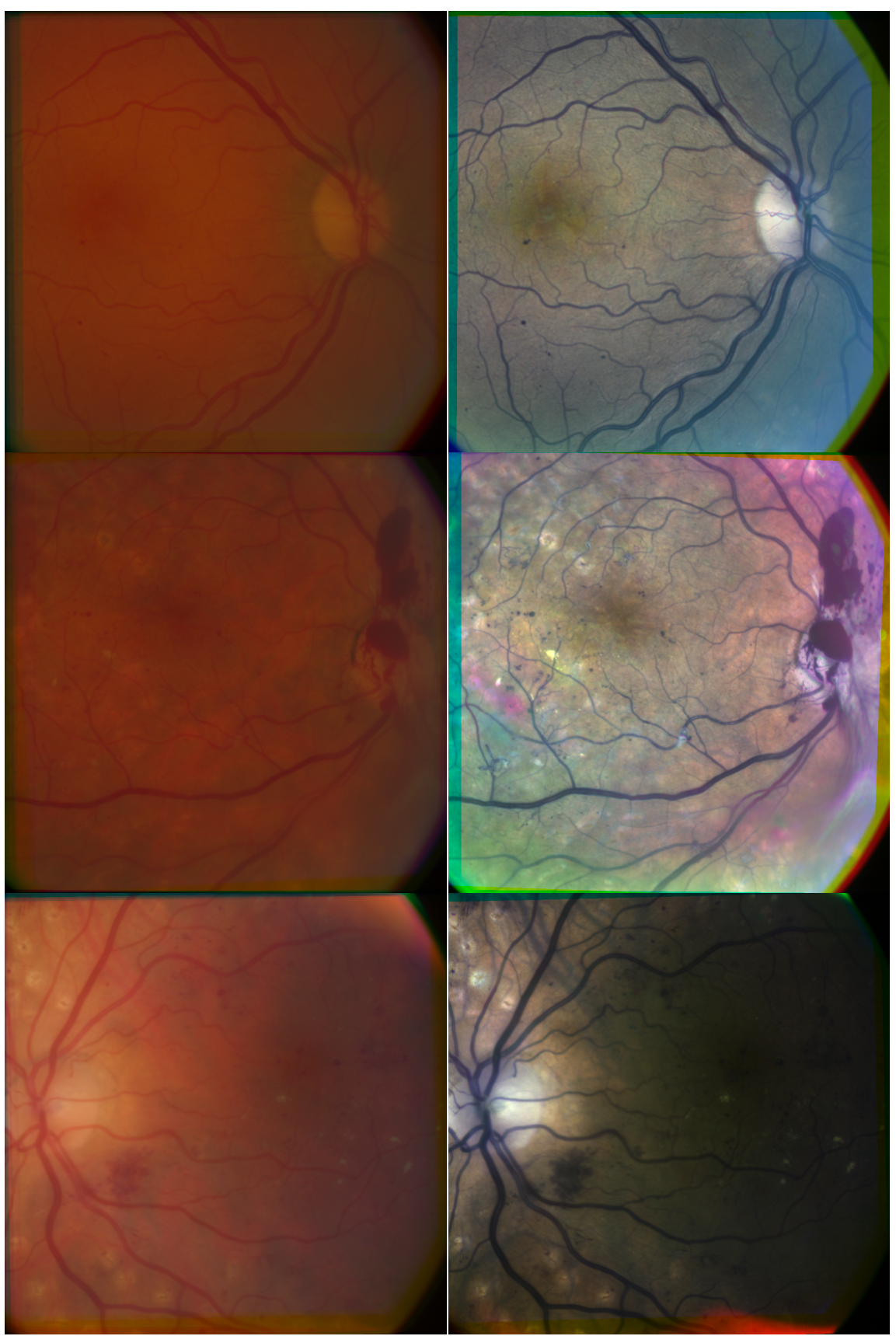

Fig. 4. RGB-images calculated from three of the 66 spectral fundus images for the CIE 1931 standard observer and D65 illumination (left column), and three-channel images the same fundi using specified registered spectral color channels (right column). No image processing (e.g. contrast enhancement) was applied to any of the images. 
Imaging of the diabetic subjects was conducted in the Department of Ophthalmology in the Kuopio University Hospital (Kuopio, Finland). The control subjects were imaged in the color research laboratory of the University of Joensuu (Joensuu, Finland). The subjects' pupils were dilated using tropicamide eye drops (Oftan Tropicamid, Santen Oy, Finland), and only one eye was imaged from each subject. The database doesn't yet contain any follow-up spectral images of individual patients.

Subject's fundus was illuminated with 30 different filtered lights and images were captured in each case. Usually, due to the light source's poor emission of violet light, the very first spectral channels contained no useful information and were thus omitted from the spectral images. Also, the age-related yellowing of the crystalline lens of the eye [21] and other obstructions (mostly cataract) played a significant role in this.

\section{Results and Discussion}

Total of 66 spectral fundus images were collected using equipment and methods descriped above. These spectral images were then saved with MATLAB to a custom file format called "spectral binary", which stores the spectral data and their wavelength range is a lossless, uncompressed form. In this study, a typical size for one spectral binary file with 27 spectral channels (the first three channels contained no information) was approx. $108 \mathrm{MB}$, and the total size of the database was approx. 7 GB.

From the spectral images, normal RGB-images were calculated for visualization (see three example images in Fig. 4, left column). Spectral-to-RGB calculations were performed for the CIE 1931 standard colorimetric observer and illuminant D65 22]. The 54 diabetes images showed typical findings for background and proliferative diabetic retinopathy, such as microaneurysms, small hemorrhages, hard lipid exudates, soft exudates (microinfarcts), intra-retinal microvascular abnormalities (IRMA), preretinal bleeding, neovascularization, and fibrosis. Due to the spectral channel image registration process, the colors on the outer edges of the images were distorted. On the right column of Fig. 4, some preliminary results of using specified spectral color channels are shown.

\section{Conclusions}

A database of spectral images of 66 human ocular fundi were presented. Also the methods of image acquisition and post-processing were described. A modified version of a standard ophthalmic fundus camera system was used with 30 narrow bandpass interference filters (400-700 $\mathrm{nm}$ at $10 \mathrm{~nm}$ intervals), a steady-state broadband light source and a monochrome digital CCD camera. Final spectral images had a $1024 \times 1024$ pixel spatial resolution and a varying number of spectral color channels (usually 27, since the first three channels beginning from 400 $\mathrm{nm}$ contained practically no information). Spectral images were saved in an uncompressed "spectral binary" format. 
The database consists of fundus spectral images taken from 54 diabetic patients demonstrating different signs and severities of diabetic retinopathy and from 12 healthy volunteers. In the future we aim to establish a full spectral benchmarking database including both spectral images and manually annotated ground truth similarly to DiaRetDB1 [4]. Due to the special attention and solutions needed in capturing and processing the spectral data, the image acquisition and data post-processing were described in detail in this study. The augmentation of the database with annotations and additional data will be future work. The database will be made public for all researchers.

Acknowledgments. The authors would like to thank Tekes - the Finnish Funding Agency for Technology and Innovation - for funding (FinnWell program, funding decision 40039/07, filing number 2773/31/06).

\section{References}

1. DRIVE: Digital Retinal Images for Vessel Extraction, http://www.isi.uu.nl/Research/Databases/DRIVE/

2. Staal, J.J., Abramoff, M.D., Niemeijer, M., Viergever, M.A., van Ginneken, B.: Ridge based vessel segmentation in color images of the retina. IEEE Trans. Med. Imag. 23, 501-509 (2004)

3. STARE: STructured Analysis of the Retina, http://www.parl.clemson.edu/stare/

4. Kauppi, T., Kalesnykiene, V., Kämäräinen, J.-K., Lensu, L., Sorri, I., Raninen, A., Voutilainen, R., Uusitalo, H., Kälviäinen, H., Pietilä, J.: DIARETDB1 diabetic retinopathy database and evaluation protocol. In: Proceedings of the 11th Conference on Medical Image Understanding and Analysis (MIUA 2007), pp. 61-65 (2007)

5. Delori, F.C., Burns, S.A.: Fundus reflectance and the measurement of crystalline lens density. J. Opt. Soc. Am. A 13, 215-226 (1996)

6. Savage, G.L., Johnson, C.A., Howard, D.L.: A comparison of noninvasive objective and subjective measurements of the optical density of human ocular media. Optom. Vis. Sci. 78, 386-395 (2001)

7. Delori, F.C.: Spectrophotometer for noninvasive measurement of intrinsic fluorescence and reflectance of the ocular fundus. Appl. Opt. 33, 7439-7452 (1994)

8. Van Norren, D., Tiemeijer, L.F.: Spectral reflectance of the human eye. Vision Res. 26, 313-320 (1986)

9. Delori, F.C., Pflibsen, K.P.: Spectral reflectance of the human ocular fundus. Appl. Opt. 28, 1061-1077 (1989)

10. Bone, R.A., Brener, B., Gibert, J.C.: Macular pigment, photopigments, and melanin: Distributions in young subjects determined by four-wavelength reflectometry. Vision Res. 47, 3259-3268 (2007)

11. Beach, J.M., Schwenzer, K.J., Srinivas, S., Kim, D., Tiedeman, J.S.: Oximetry of retinal vessels by dual-wavelength imaging: calibration and influence of pigmentation. J. Appl. Physiol. 86, 748-758 (1999)

12. Ramella-Roman, J.C., Mathews, S.A., Kandimalla, H., Nabili, A., Duncan, D.D., D'Anna, S.A., Shah, S.M., Nguyen, Q.D.: Measurement of oxygen saturation in the retina with a spectroscopic sensitive multi aperture camera. Opt. Express 16, 6170-6182 (2008) 
13. Khoobehi, B., Beach, J.M., Kawano, H.: Hyperspectral Imaging for Measurement of Oxygen Saturation in the Optic Nerve Head. Invest. Ophthalmol. Vis. Sci. 45, 1464-1472 (2004)

14. Hirohara, Y., Okawa, Y., Mihashi, T., Amaguchi, T., Nakazawa, N., Tsuruga, Y., Aoki, H., Maeda, N., Uchida, I., Fujikado, T.: Validity of Retinal Oxygen Saturation Analysis: Hyperspectral Imaging in Visible Wavelength with Fundus Camera and Liquid Crystal Wavelength Tunable Filter. Opt. Rev. 14, 151-158 (2007)

15. Hammer, M., Thamm, E., Schweitzer, D.: A simple algorithm for in vivo ocular fundus oximetry compensating for non-haemoglobin absorption and scattering. Phys. Med. Biol. 47, N233-N238 (2002)

16. Styles, I.B., Calcagni, A., Claridge, E., Orihuela-Espina, F., Gibson, J.M.: Quantitative analysis of multi-spectral fundus images. Med. Image Anal. 10, 578-597 (2006)

17. Johnson, W.R., Wilson, D.W., Fink, W., Humayun, M., Bearman, G.: Snapshot hyperspectral imaging in ophthalmology. J. Biomed. Opt. 12, 014036 (2007)

18. Stewart, C.V., Tsai, C.-L., Roysam, B.: The dual-bootstrap iterative closest point algorithm with application to retinal image registration. IEEE Trans. Med. Imag. 22, 1379-1394 (2003)

19. Yang, G., Stewart, C.V., Sofka, M., Tsai, C.-L.: Registration of challenging image pairs: initialization, estimation, and decision. IEEE Trans. Pattern Anal. Mach. Intell. 29, 1973-1989 (2007)

20. MATLAB: MATrix LABoratory, The MathWorks, Inc., http://www.mathworks.com/matlab

21. Gaillard, E.R., Zheng, L., Merriam, J.C., Dillon, J.: Age-related changes in the absorption characteristics of the primate lens. Invest. Ophthalmol. Vis. Sci. 41, 1454-1459 (2000)

22. Wyszecki, G., Stiles, W.S.: Color Science: Concepts and Methods, Quantitative Data and Formulae, 2nd edn. John Wiley \& Sons, Inc., New York (1982) 